\title{
EFFICIENT IMAGE RETRIEVAL BY EXAMPLES
}

\author{
Roberto Brunelli \\ ITC-irst \\ Via Sommarive 18, I-38050 Povo (TN), ITALY \\ brunelli@itc.it
}

Ornella Mich

ITC-irst

Via Sommarive 18, I-38050 Povo (TN), ITALY

mich@itc.it

\begin{abstract}
A currently relevant research field in information sciences is the management of non-traditional distributed multimedia databases. Two related key issues are achieving an efficient content-based query by example retrieval and a fast response time. This paper presents the architecture of a distributed image retrieval system which provides novel solutions to these key issues. In particular, a way to quantify the effectiveness of low level visual descriptors in database query tasks is presented. The results are then used to improve the system response time, an important issue when querying very large databases. A new mechanism to simplify user queries, featuring local modification of the comparison metric in the space of image descriptors, is presented and discussed.
\end{abstract}

Keywords: image similarity retrieval, feature extraction, image-based queries, distributed databases, clustering.

\section{INTRODUCTION}

The current ever growing amount of multimedia data requires a big integrated effort in the research fields of Computer Vision, Information Retrieval and Database Management for its effective management. In particular, retrieving information from multimedia repositories requires the development of techniques to supplement traditional methods based on textual descriptions and searches. The reason for this necessity is twofold: associating textual descriptions to multimedia data can be very 
expensive, and, what is even more important, textual descriptions may not characterize data adequately for subsequent retrieval. The latter issue is of particular relevance for multimedia material, whose searching criteria and features are highly dependent on user goals.

An attempt to overcome this limitation is through query by example where non textual queries are formulated by the user using multimedia items related to the material he/she is looking for (e.g. images or video clips for searching footage). Recently many multimedia retrieval systems have investigated the query by example framework. Some of the most relevant are QBIC ${ }^{(\mathrm{TM})}$, the IBM's Query By Image Content system ( Flickner et al., 1995), Excalibur Visual RetrievalWare ${ }^{(\mathrm{r})}$, a comprehensive application development software to provide content-based, highperformance retrieval for multiple types of digital visual media, Visual Information Retrieval (VIR) Image Engine by Virage, a set of libraries for analyzing and comparing the visual content of images, MARS: Multimedia Analysis and Retrieval System (Porkaew et al., 1999), an application developed by the Beckman Institute and Department of Computer Science at the University of Illinois, whose aim is to integrate various techniques in the fields of Image Processing and Information Retrieval into an Image Data Base Management System that is accessible from the web.

In the query by example framework, the user formulates a query by providing examples of objects similar to the one he/she wishes to retrieve. The system converts them into an internal representation used for assessing their similarity to the items stored in the database to be searched. The main advantage of query by example is that the user is not required to provide an explicit description of the items which is instead computed by the system. In order for this paradigm to be effective, good content descriptions must be computed automatically by the system and ways to compare them obtaining results in accordance with human judgements should also be available.

This paper discusses the use of pattern analysis techniques, such as density estimation and clustering, and multidimensional scaling, for the development of a computer assisted image search system. The architecture of the system is described in Section 2. The issue of small yet effective image descriptors is considered in Section 3, while relevance feedback and query optimization are described in Section 4 and Section 5 respectively. Some concluding remarks are reported in Section 6 . 


\section{SYSTEM ARCHITECTURE}

The structure of an image retrieval system to support the query by example paradigm for multiple distributed databases is presented in Figure 1. The system is configured as a client-server architecture in which a client application can submit a user query to multiple image servers. The answers from multiple image servers are then merged and proposed to the user as a single result.

Following the query by example paradigm, users rely on the images themselves to formulate queries. A generic image $\mathcal{I}$ is characterized as a triple $(\boldsymbol{I}, \boldsymbol{F}, \boldsymbol{M})$ whose elements represent a complete description of the image pixels $\boldsymbol{I}$, possibly indirectly by pointing to the corresponding memory storage, a derived feature description $\boldsymbol{F}=\left\{\boldsymbol{F}_{i}\right\}$, automatically computed by the system, and associated meta data $\boldsymbol{M}$ providing information on image contents. Derived image descriptions can be computed directly by the client application while meta information is not usually provided automatically.

A query by example $\boldsymbol{Q}$ is defined by giving a set $\boldsymbol{E}$ of images and, possibly, by selecting a subset $\boldsymbol{f}$ of $\boldsymbol{F}$ and a comparison strategy $\boldsymbol{S}$ to be used by the image servers when comparing the query images to those stored in the database:

$$
Q=(E, f, S)
$$

The query images can be

- local or remote images accessible from the client application: the user may provide appropriate meta-data which can be used to supplement the visual similarity search with a more traditional textual search;

- images from a previous query considered as relevant by the user;

- images from selected image servers, relying on the browsing functionalities of the client.

In order to answer a query, the image server compares the images in the query set $\boldsymbol{E}$ to the stored ones using strategy $\boldsymbol{S}$, obtaining a dissimilarity score for each of them. The dissimilarity of images could be computed using both the derived descriptors $f$ and image meta-data $M$. Derived decriptors are often represented as numerical vectors while meta data are usually in textual form. The analysis presented in this paper will be limited to the use of derived descriptors represented as numerical vectors, leaving out any available meta data in the computation of image similarity. 
As the set of query images must be compared to other images, a function to compute the dissimilarity of an image from an image set must be introduced. If we restrict to metric spaces for the derived descriptors, the distance between an image $I$ and an image set $\boldsymbol{E}$ can be computed using the following formula:

$$
D(\mathcal{I}, \boldsymbol{E})=\min _{\mathcal{I}^{\prime} \in \boldsymbol{E}} d\left(\mathcal{I}, \mathcal{I}^{\prime}\right)
$$

where $d$ represent the distance defined in the metric space.

The effectiveness of feature comparison is improved by the use of relevance feedback which modifies the distance of the metric space using information derived from the interaction of the user with the system. The servers then sort database items by increasing dissimilarity. The set $\boldsymbol{A}$ of the top ranked ones is returned to the client together with their dissimilarity value and, if available and requested, associated metadata. The client, upon receiving the answer from each server, sorts the resulting complete set by dissimilarity and offers to the user a single answer.

\section{IMAGE DESCRIPTION}

One of the key issues in querying image databases by similarity is the choice of appropriate image descriptors and corresponding similarity measures. In a recent paper (Brunelli and Mich, 1999) the problem of quantifying the effectiveness of several low level visual descriptors was addressed. The proposed solution relies on the following definitions:

Definition 1 Given an n-dimensional histogram space $\mathcal{H}$ and a dissimilarity measure $d$ on $\mathcal{H}$, the capacity curve $C$ of $\mathcal{H}$ is defined as the density distribution of the dissimilarity between the two elements of all possible histogram couples within $\mathcal{H}$.

Histogram capacity curves provide a basis on which the effectiveness, i.e. the discrimination ability, of different image descriptors can be compared. The shape of $C(t)$ is an indicator of the distribution of histograms in $\mathcal{H}$ with the topology induced by the selected comparison dissimilarity measure. If the average value of dissimilarity is low, histograms are not sparse enough in $\mathcal{H}$ and histogram indexing is not effective. This can be formalized by the following definition:

Definition 2 The indexing effectiveness $\mathcal{E}$ of an histogram space $\mathcal{H}$ is given by the average dissimilarity value:

$$
\mathcal{E}=\int y C(y) d y
$$




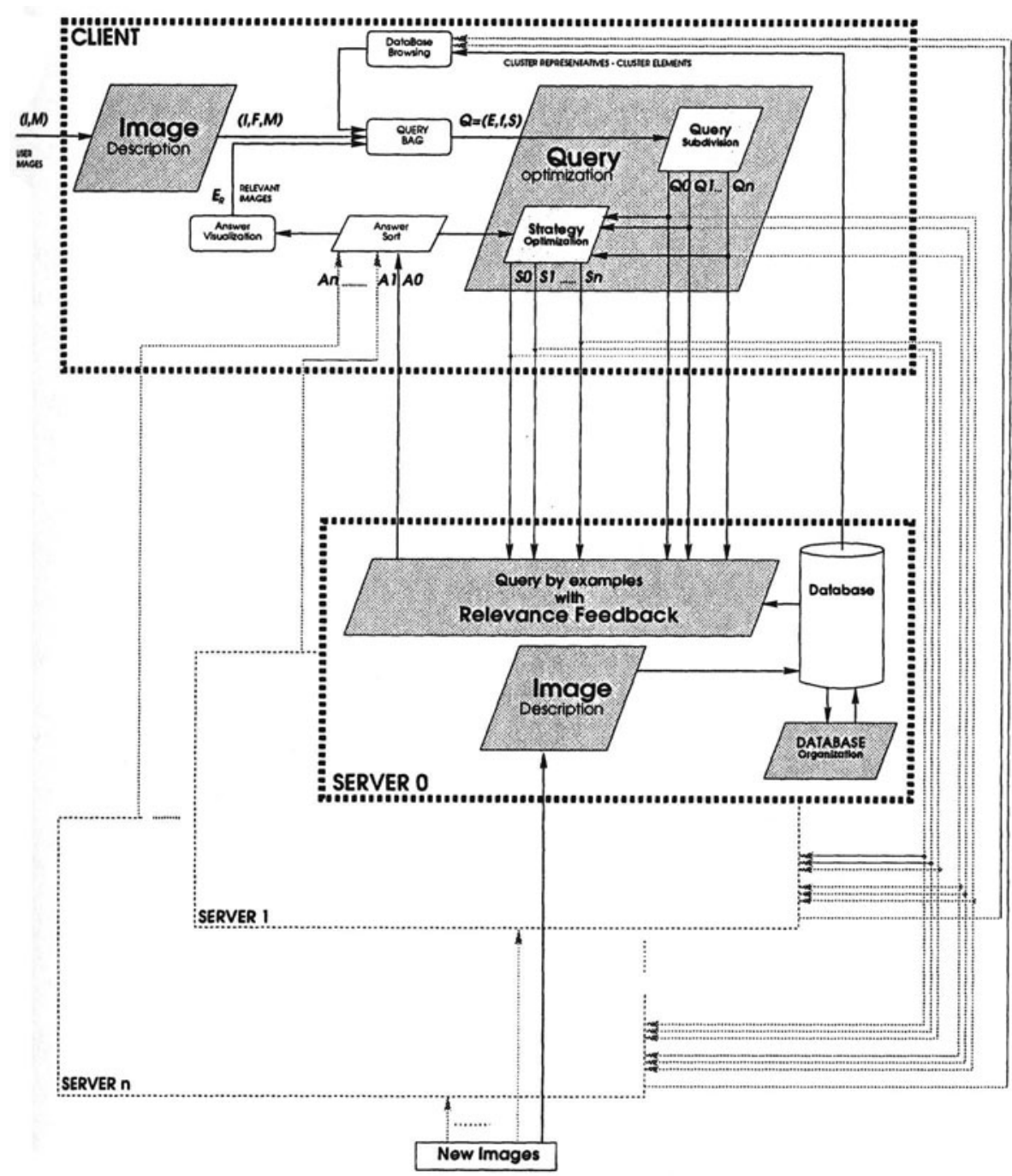

Figure 1 A general architecture for an image retrieval system based on the query by example paradigm. The shaded blocks are considered in detail by the current paper.

The indexing effectiveness $\mathcal{E}$ can be used to assess the performance several descriptor-dissimilarity combinations for image retrieval applications. Several ways to compute image dissimilarity were considered in (Brunelli and Mich, 1999): $\chi^{2}$, Kolmogorov-Smirnov, Kuiper, and $L_{p}$ norms. The $L_{1}$ norm provided the best overall results in terms of indexing effectiveness and stability with respect to the number of histogram bins used. A further benefit of using the $L_{1}$ norm is that it can be efficiently computed using parallel instructions available on current 
personal computers. Usage of these instructions results in a four-times increase in the speed at which image distances can be computed, making the comparison of a million images per second a feasible task. The main findings of (Brunelli and Mich, 1999) on the discrimination ability of the following basic image descriptors:

- hue, $H$ : a scalar descriptor which associates to an $(r, g, b)$ triple representing the pixel color its tint; the resulting density represents a circular variable;

- luminance, $I$ : a scalar descriptor which associates to an $(r, g, b)$ triple representing the pixel color the normalized sum of its components;

- edgeness, $E$ : the magnitude of the gradient $\sqrt{\left(\partial_{x} I\right)^{2}+\left(\partial_{y} I\right)^{2}}$ where $I$ represents the image luminance;

- hue co-occurrence: space $\boldsymbol{S}$ is partitioned into couples of pixels by means of a binary spatial relation: a pixel located at $(x, y)$ is associated to a pixel at $(x+\Delta x, y+\Delta y)$ and the tints of the two pixels are used as indices in a 2-dimensional histogram;

- luminance co-occurrence: the same as hue co-occurrence using pixel luminances.

are summarized in Table 1 and Figure 2. Reported data are based on two different image sets:

- VIDEO: a set of 40000 frames from nine different video clips. The video material was varied, ranging from comics, news, to documentaries and action movies.

- STILLS: a set of 3500 still images from a commercial collection, providing more colorful and high quality images than the average video material of the above database.

The effectiveness of the different descriptors can also be used to optimize the order in which they are compared. In image retrieval tasks, a threshold on the minimum acceptable similarity is usually imposed to limit the number of retrieved items. The computation of image dissimilarity using the $L_{1}$ norm can be stopped as soon as its monotonically increasing value exceeds the retrieval threshold. When multiple histograms are used to characterize an image, they can be concatenated in many different ways to obtain a single numerical vector describing the image. However the order used does matter. Comparing the descriptors 
sorted by decreasing effectiveness is expected to increase the computational savings associated to the use of a retrieval threhsold. Experiments on the same data used in (Brunelli and Mich, 1999) are reported in Figure 3 and confirm this expectation.

Table 1 The effectiveness $\mathcal{E}$ of some low-level visual descriptors.

\begin{tabular}{lrr}
\hline Descriptor & $\mathcal{E}_{\text {VIDEO }}$ & $\mathcal{E}_{\text {STILLS }}$ \\
\hline Co-occurrence (hue) & 57 & 68 \\
Hue & 55 & 70 \\
Co-occurrence (lum) & 52 & 50 \\
Luminance & 43 & 46 \\
Edgeness & 22 & 32 \\
\hline
\end{tabular}

\section{QUERY BY EXAMPLES WITH RELEVANCE FEEDBACK}

Relevance feedback is a fundamental mechanism by which system response can be improved by using information fed by the user (Cox et al., 1996; Ishikawa and Faloutsos, 1998; Sclaroff et al., 1997; Porkaew et al., 1999). Whenever the system presents to the user a set of images considered to be similar to the provided examples, the user can pick among them the images he/she considers most relevant to the submitted query and add them to the original query. The resulting extended set $\boldsymbol{E}_{R}$ can be used to improve system response in a variety of ways (Porkaew et al., 1999). A common approach to the implementation of relevance feedback for a system using image descriptors in numerical form is that of feature weighting and is based on the vector model used for textual documents.

Image derived descriptors $\boldsymbol{F}=\left\{\boldsymbol{F}_{i}\right\}$ are obtained by binning, with the same number of bins, the density estimates of the corresponding image characteristics (e.g. luminance, hue, etc.). Exploiting the homogeneity of descriptors normalization and dimensionality, the dissimilarity of two images can be computed by:

$$
d\left(\mathcal{I}, \mathcal{I}^{\prime}\right)=\sum_{i} \alpha_{i} \sum_{j} w_{i j}\left|F_{i j}-F_{i j}^{\prime}\right|
$$

where $i$ represents the $i$-th descriptor and $j$ the value of the $j$-th bin of the descriptor. This distance introduces a metric structure in the derived descriptors space and can be used to compute the distance of the query set $\boldsymbol{E}_{R}$ from each database item using the formula reported in Eq. 2. 


\section{Image descriptors capacity curves}

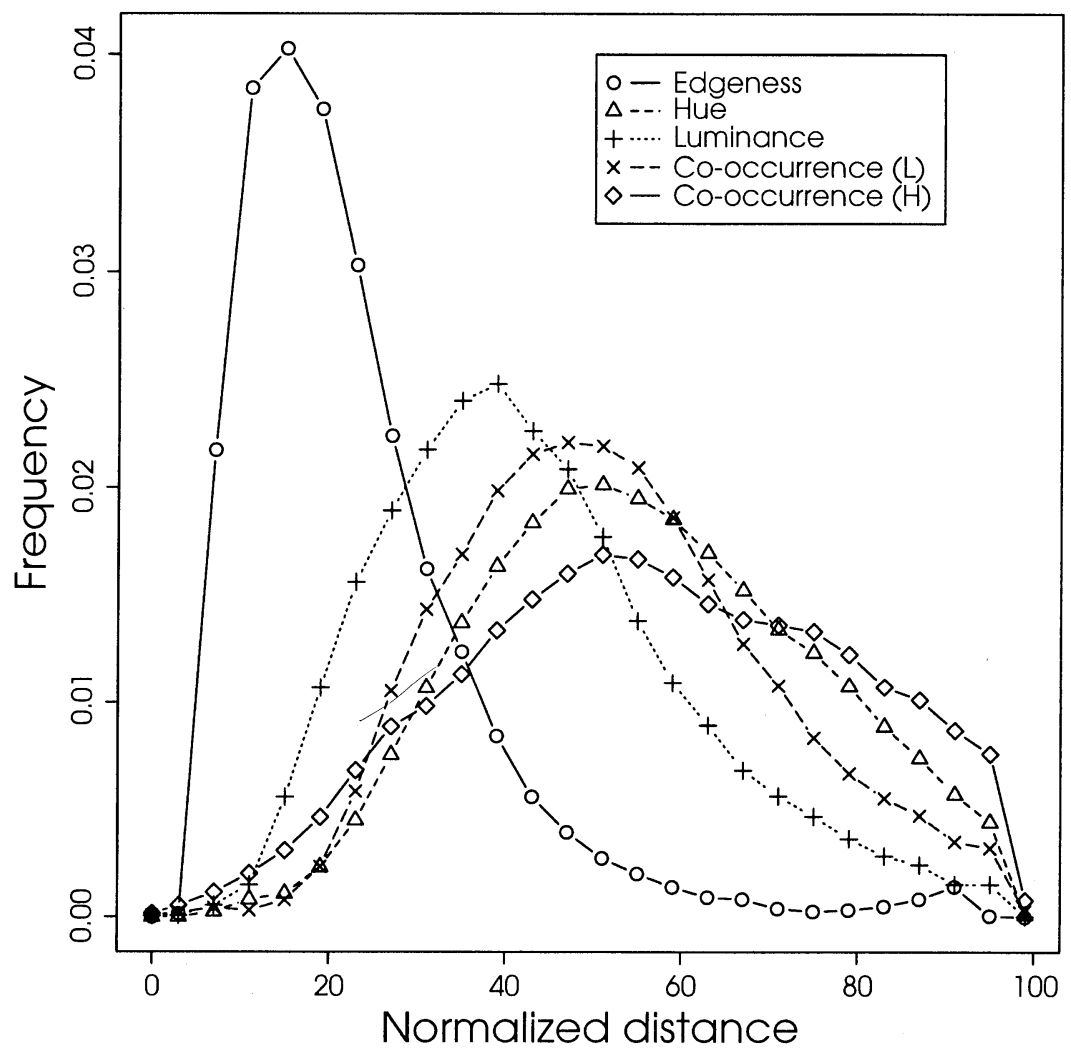

Figure 2 The plots report the capacity curves, computed using the $L_{1}$ distance and 64 bins per histogram, for the image descriptors described in the text.

The default set $\left\{\alpha_{i}\right\}$ is $\{1, \ldots, 1\}$ and can be modified by the user to assign different weights to the image descriptors, possibly excluding some of them from the computation of $d$. The set $\left\{w_{i j}\right\}$ is computed by the system and is used to incorporate relevance feedback into the comparison metric. Relevant images should be similar to each other for some of the components of their descriptors $F_{i j}$. This means that the standard deviations $\sigma_{i j}$ computed over set $\boldsymbol{E}_{R}$ should be small for the components capturing the similarity of the images and larger for the components which are not relevant. A method to emphasize distances along the relevant directions is to use the following set of weights (Rui et al., 


\section{Effect of descriptors ordering on retrieval efficiency}

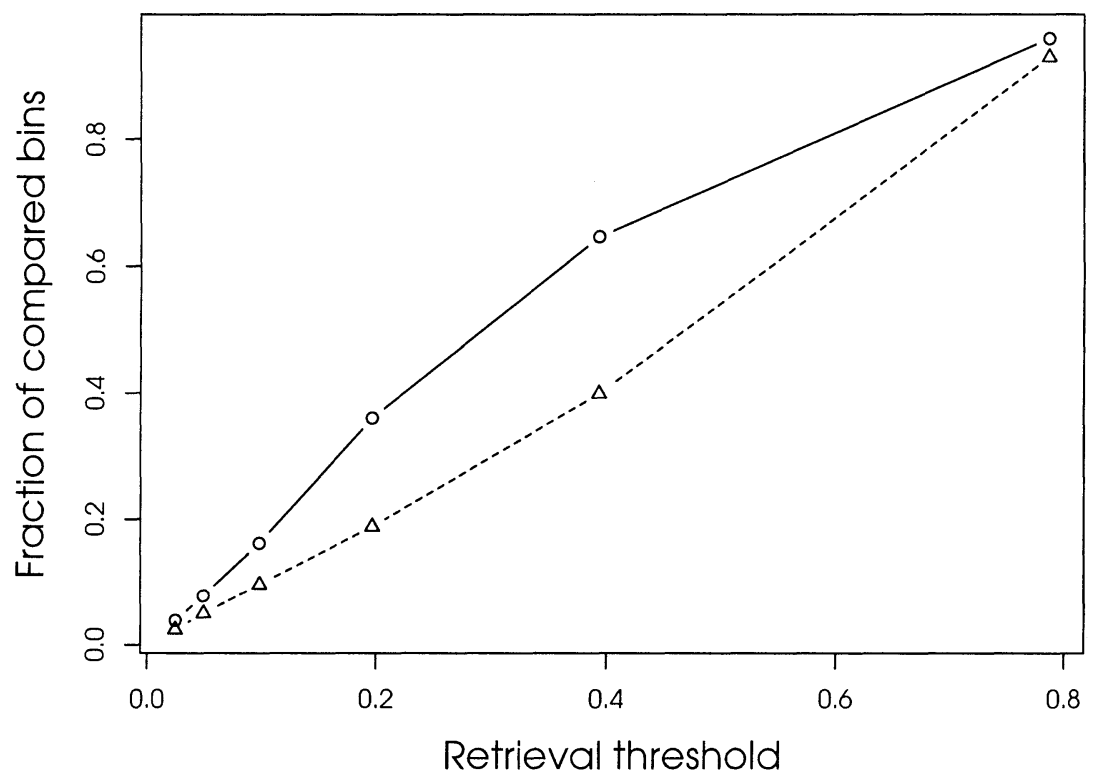

Figure 3 The plot reports (in double logarithmic scale) the expected gain in speed resulting from properly sequencing the image descriptors before comparing them. Note the significant advantage over the worst case, where the order in which the descriptors are used is inversely proportional to their capacity.

1997):

$$
w_{i j}=k \frac{1}{\sigma_{i j}}
$$

In this paper a family of weighting schemes is derived from the previous equation

$$
w_{i j}=k_{\beta} \frac{1}{\sigma_{i j}}
$$

$k_{\beta}=\left(\sum_{l m} \sigma_{l m}^{-\beta}\right)^{-1}$ is a normalizing factor, while $\beta$ is a parameter which modulates the weighting effect and can be varied to optimize image comparison results. The effect of feature weighting on the computation of distances in descriptor space is to increase the value of distances along 
the directions of minimal variance of the query set. There are some major drawbacks to the use of Equation 6:

- the use of $\sigma_{i j}$ tacitly assumes that the images in the query represent a compact set with ellipsoidal shape;

- the comparison metric is modified in the same way all over the descriptor space;

Furthermore, the amount of weighting specified by $\beta$ is expected to be query dependent and should be optimized on a case-by-case basis. A way to overcome these drawbacks is presented in the following section.

\section{QUERY OPTIMIZATION}

The effect of the drawbacks associated to the use of Equation 6 on the effectiveness and efficiency of relevance feedback can be minimized by determining whether the specified query set $\boldsymbol{Q}$ while not being compact itself, is composed by two or more compact sets: the query could then be split into simpler subqueries, each of them better suited to the use of Equation 6. Let us note that this splitting also introduces local modification in the metric structure of the descriptors space.

The cloud of points representing the query images in the descriptor space may exhibit local grouping, i.e. clusters, suggesting the splitting of the original query set into multiple subsets, each of them characterized by the images belonging to one of the clusters.

From a data analysis perspective, the relevant issue is whether the structure of the point distribution supports the presence of multiple clusters or not. There are no completely satisfactory methods to determine the number of clusters for any type of cluster analysis (Milligan and Cooper, 1985; Jain and Moreau, 1987). The situation analysed by the current paper presents additional difficulties due to the small number of images used to define the query: no asymptotic results can be used, and methods relying on density estimates can not be applied. The chosen strategy is based on two steps:

1 establish whether the original query should be split or not;

2 if the original query should be split determine the number of clusters into which it should be split.

The first step is based on the use of a statistic originally proposed by Duda and Hart (Duda and Hart, 1973). Let us denote with $d\left(\mathcal{I}, \mathcal{I}^{\prime}\right)$ the distance between the descriptors of two images $\mathcal{I}, \mathcal{I}^{\prime}$ and with $J(c)$ the clustering criterion function for $c$ clusters $C_{1}, \ldots, C_{c}$ : 


$$
J(c)=\sum_{i=1}^{c} \sum_{\mathcal{I} \in C_{i}} d\left(\mathcal{I}, \boldsymbol{m}_{i}\right)
$$

where $\boldsymbol{m}_{i}$ is the central image of the $i$-th cluster. The quantity $J(c)$ is a random variable whose average value decreases monotonically with $c$. In particular, if data are organized into $\hat{c}$ compact, well separated clusters, the value of $J(c)$ is expected to decrease rapidly until $\hat{c}=c$, and much more slowly thereafter. Knowledge of the distribution of $J(2) / J(1)$ under the null hypothesis that all samples belong to a single cluster forms the basis for a test to reject or accept the null hypothesis. Unfortunately, analytical results are often not available. An approximate result is derived in (Duda and Hart, 1973) when the distance used in the comparison is the Euclidean norm. As the comparison metric considered in this paper is the $L_{1}$ norm for which results are much harder to obtain, a Monte Carlo approach was chosen (Dubes, 1987). As detailed in Section 3 , each image is represented by histograms of several low level visual features, normalized to unit. In order to determine the distribution of $\mathcal{J}=J(2) / J(1)$ for different sample sizes $n$ (from 6 to 16), 10000 random samples were generated that satisfied the image descriptors constraints: number of features, number of bins, and normalization to unit. For each random sample the Linde-Buzo-Gray clustering algorithm (Linde et al., 1980) using the $L_{1}$ metric was applied 10 times to find the optimal two cluster partition. The corresponding values of $\mathcal{J}$ were then used to compute the required distributions which are summarized in Figure 4. As anticipated, the distributions for different values of $n$ are markedly different, $n$ being too small to ensure an asymptotic regime. Given a set of $N$ query images the value of $\mathcal{J}$ is computed: if the null hypothesis of a single cluster can be rejected with the prescribed confidence, the appropriate number of clusters should then be determined. The most appropriate number of subqueries into which the original query should be split is determined by the so called silhouette coefficient $\mathcal{S}$ introduced in (Kaufman and Rousseeuw, 1990). Let us introduce the following quantities:

$$
\begin{aligned}
a(i) & =\frac{1}{n_{C(i)}} \sum_{j \in C(i), j \neq i} d\left(\mathcal{I}_{i}, \mathcal{I}_{j}\right) \\
\Delta(i, C) & =\frac{1}{n_{C}} \sum_{j \in C} d\left(\mathcal{I}_{i}, \mathcal{I}_{j}\right) \\
b(i) & =\min _{C \neq C(i)} \Delta(i, C)
\end{aligned}
$$




\section{$J(2) / J(1)$ Statistic for L1 clustering}

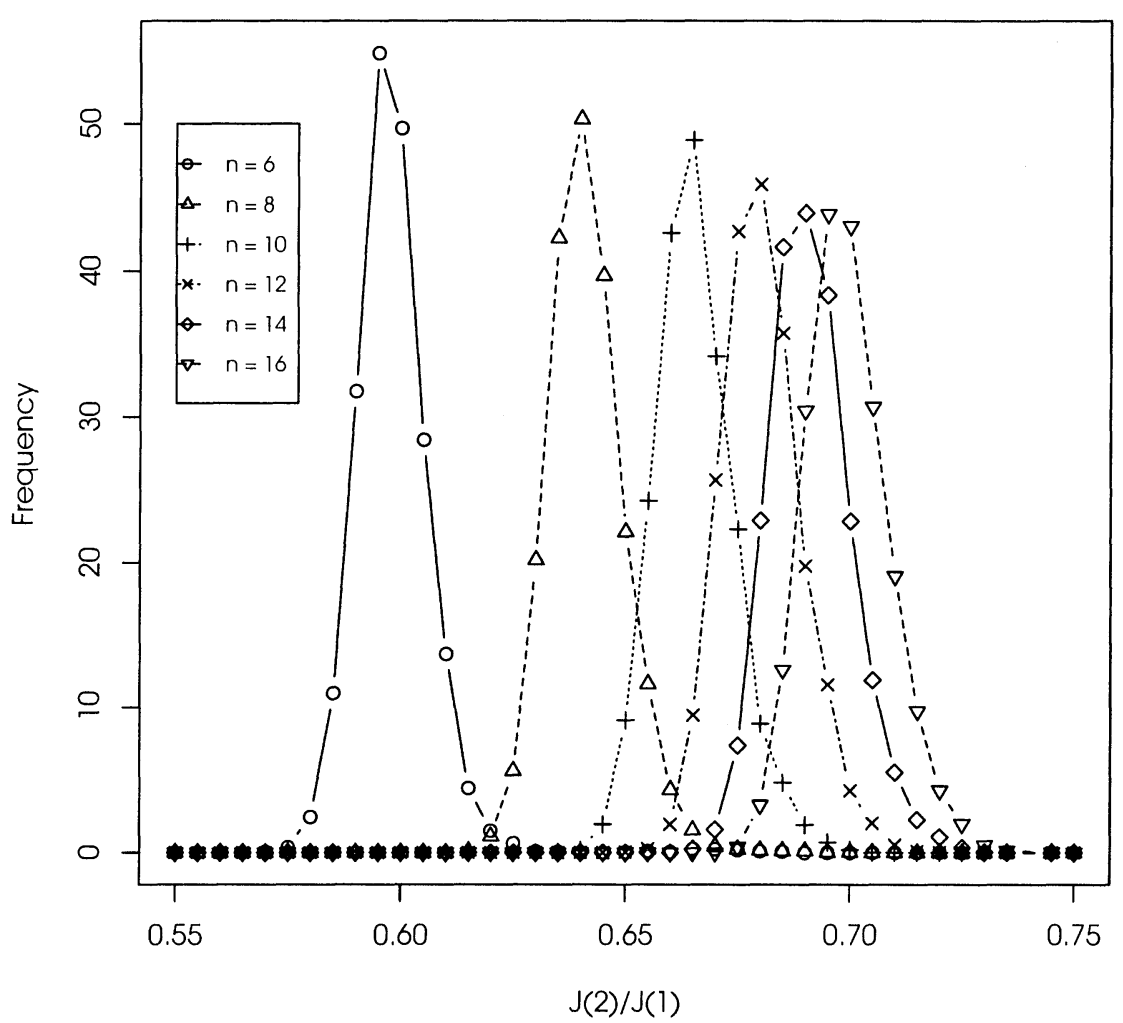

Figure 4 The plot reports the distribution of the $J(2) / J(1)$ statistic for $L_{1}$ clustering using sample points generated taking into account the characteristics of the image descriptors used in reported experiments.

where $n_{C}$ is the number of elements in cluster $C$; the silhouette of element $i$ is then defined as

$$
s(i)=\frac{b(i)-a(i)}{\max \{a(i), b(i)\}}
$$

When a cluster contains a single object, $s(i)=0$. The higher the value of $s(i)$ the stronger the membership of $i$ to its corresponding cluster. Elements that can not be clearly assigned to any cluster have a silhouette value near to zero. The silhouette coefficient $\mathcal{S}$ is then defined as

$$
\mathcal{S}=\frac{1}{N} \sum_{i=1}^{N} s_{i}
$$


The value of $\mathcal{S}$ is bound to the closed interval $[-1,1]$ : the higher the value the better the overall classification of data for the given clustering. Furthermore, $\mathcal{S}$ is a dimensionless quantity that does not change when the distances between samples are multiplied by a constant factor. The knowledge of the silhouette coefficient can be used to choose an appropriate number of clusters $\hat{k}$ so that

$$
\mathcal{S}(\hat{k})=\max _{k=2, \ldots, K} \mathcal{S}(k)
$$

The above computations are used to subdivide the original query images into several, simpler queries, each of which is better conditioned for the application of relevance feedback mechanisms (see Figure 5 and Figure 6) . The resulting simplified queries are then submitted to the image databases. For each simplified query a new comparison metric is computed according to Eq. 6. As a result, the metric used for image comparison is no longer a uniform modification of the original $L_{1}$ distance: each subquery locally modifies the comparison metric, overcoming one of the limitations of the original feature weighting approach.

As previously noted, the value of $\beta$ used in Eq. 6 can be tuned to each query to increase the effectiveness of image retrieval. This can be done using information derived from the interaction of the user with the system. During his/her interaction with the system the user selects some of the images presented by the system as relevant to the current query and adds them to the query set. At each interaction, the system returns an image set $\boldsymbol{A}$ with the $N$ database images most similar to the submitted query. The rank of the relevant images in the sorted lists returned by the system can be used to determine an optimal value of $\beta$. Let us restrict to a discrete set $\left\{\beta_{j}\right\}$ of possible values. For each value $\beta_{j}$ several synthetic queries can be performed on $\boldsymbol{A}$ by excluding for each of the queries one of the images of $\boldsymbol{E}_{R}$ which belongs to one of the queried databases. Due to the way the distance of each database image from $\boldsymbol{E}_{R}$ is computed, this ensures that the excluded image belongs to $\boldsymbol{A}$ (as its distance from the query set is zero). The average rank of the excluded images over the synthetic queries is then used for the optimization: the value of $\beta$ providing the lowest average rank defines the comparison metric. Some examples are reported in Fig. 7.

\section{CONCLUSION}

In this paper an architecture for a general image retrieval system featuring relevance feedback was presented and discussed. Several low level image descriptors (hue, luminance, etc.) have been compared and their retrieval effectiveness assessed through their capacity curves. The re- 


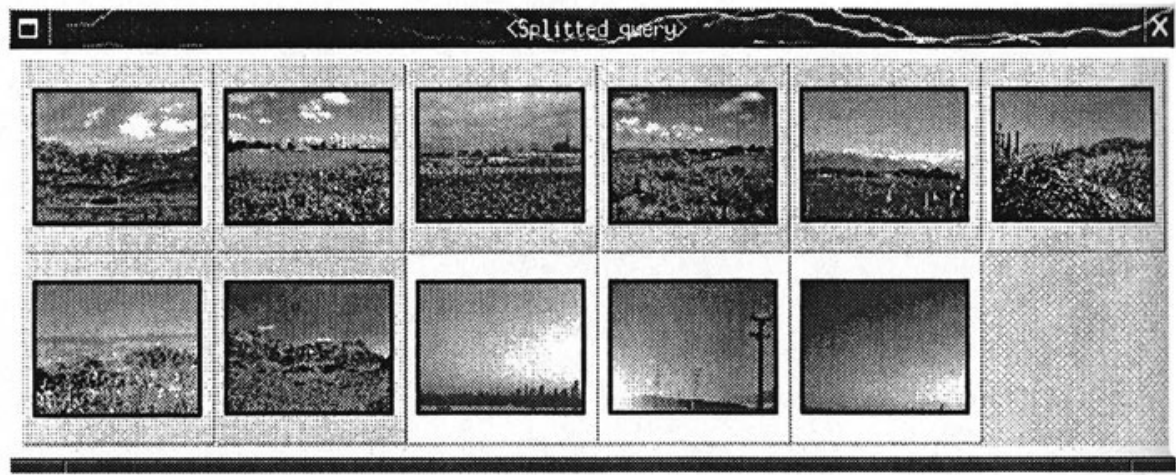

\section{Splitting criteria}

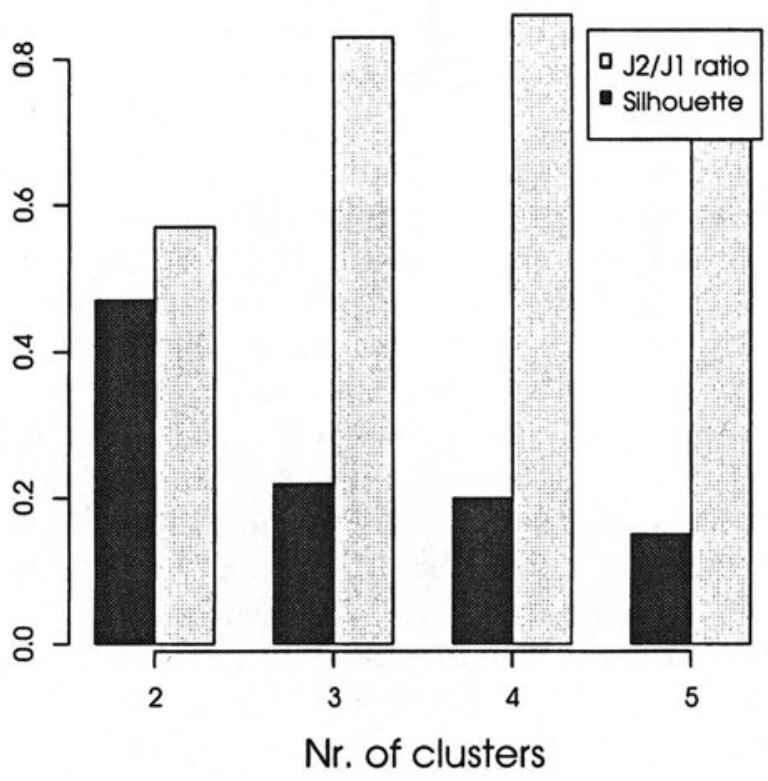

Figure 5 The upper figure presents a complex query-by-example with 11 images. The value of the $J 2 / J 1$ ratio suggests that the original query should be split, while the computed silhouette coefficients suggest that the optimal number of clusters is two. The resulting sub-queries are identified by a different shading of the frames. Note that the 8 images grouped in the first sub-query exhibit significant variation, yet the algorithm has correctly grouped them. 


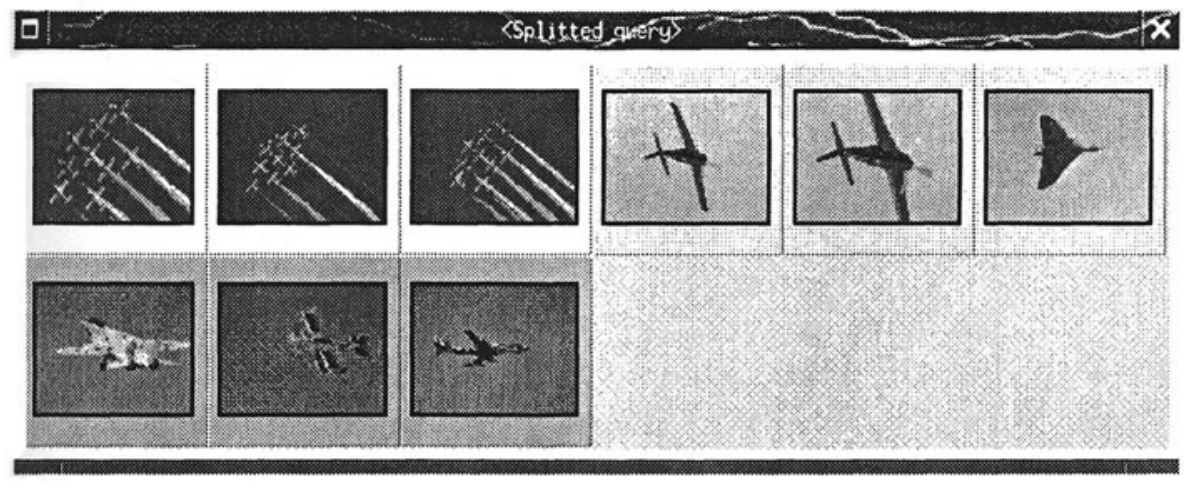

\section{Splitting criteria}

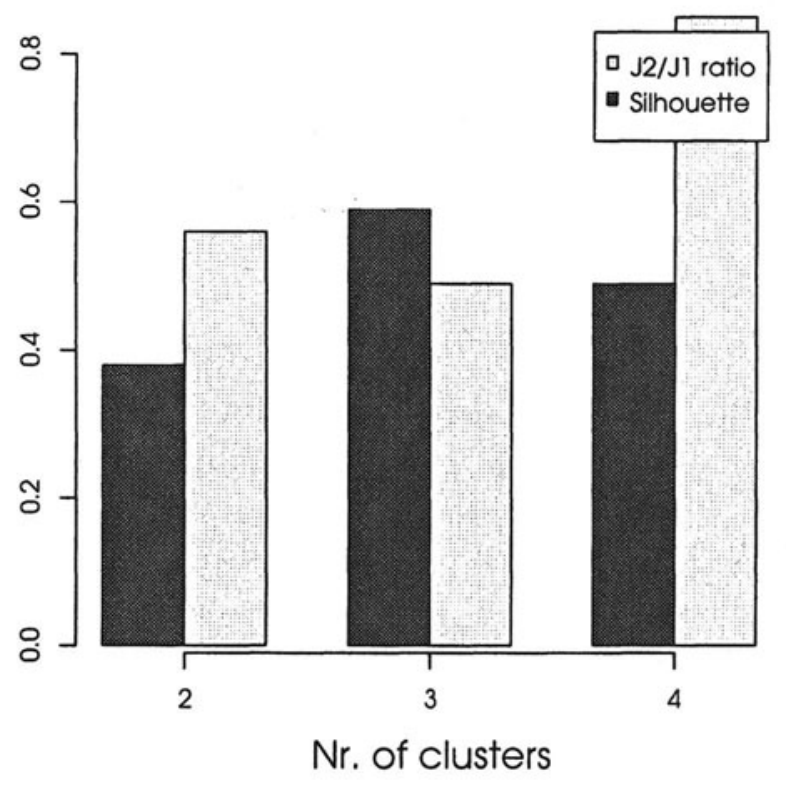

Figure 6 Another example of a complex query that would be split by the presented algorithm. The value of the $J 2 / J 1$ ratio suggests that the original query should be split, while the computed silhouette coefficients suggest that the optimal number of clusters is three. The resulting sub-queries are identified by a different shading of the frames. 
Optimal choice of $\beta$

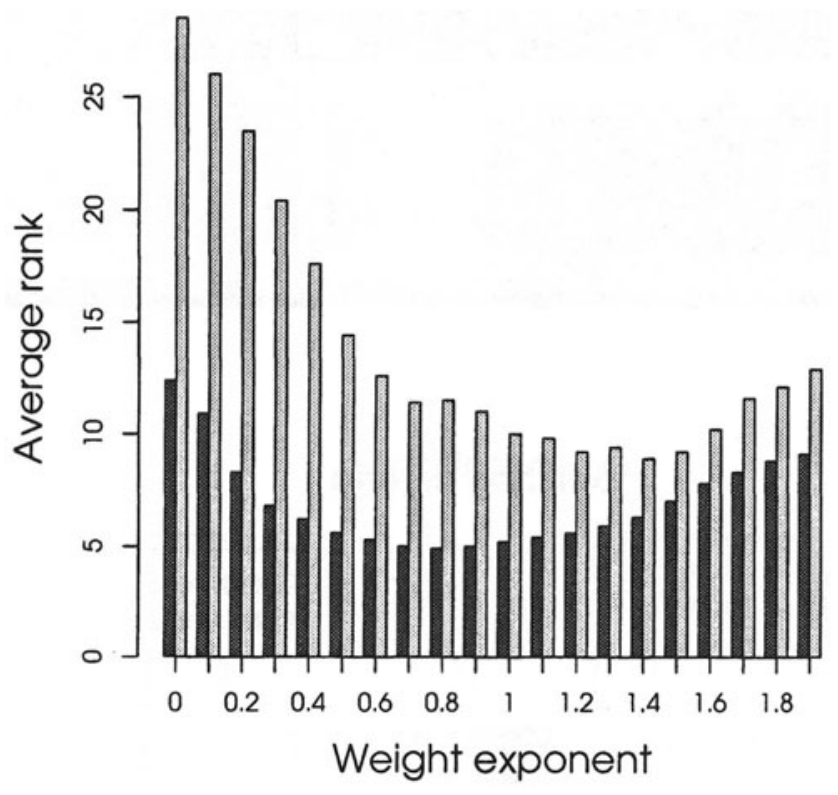

Figure 7 The plot reports the average rank distribution for different values of $\beta$ from two sample cases.

sults permit the design of scalable image retrieval systems which make optimal use of computational and storage resources. A novel approach to relevance feedback has also been presented. In particular, the possibility of tuning search strategies and comparison metrics to varying user behaviour was investigated and novel solutions were presented using pattern analysis techniques.

\section{Notes}

1. In this context, a dissimilarity measure is a bounded, positive, and symmetric function defined over a subset of $\mathbf{R}^{\mathbf{n}} \times \mathbf{R}^{\mathbf{n}}$. 


\section{References}

Brunelli, R. and Mich, O. (1999). On the Use of Histograms for Image Retrieval. In Proceedings of ICMCS'99, Florence.

Cox, I., Miller, M., Omohundro, S., and Yianilos, P. (1996). PicHunter: Bayesian Relevance Feedback for Image Retrieval. In Proc. of Int. Conf. on Pattern Recognition, Austria.

Dubes, R. C. (1987). How many clusters are best? - An experiment. Pattern Recognition, 6(20):645-663.

Duda, R. O. and Hart, P. E. (1973). Pattern Classification and Scene Analysis. Wiley, New York.

Flickner, M., Sawhney, H., Niblack, W., Ashley, J., Huang, Q., Dom, B., Gorkani, M., Hafner, J., Lee, D., Petkovic, D., Steele, D., and Yanker, P. (1995). Query by Image and Video Content: The QBIC System. Computer, pages 23-32.

Ishikawa, Y. and Faloutsos, R. S. C. (1998). MindReader: Querying Databases through Multiple Examples. In Proceedings of the International Conference on Very Large Data Bases.

Jain, A. K. and Moreau, J. V. (1987). Bootstrap technique in cluster analysis. Pattern Recognition, 20(5):547-568.

Kaufman, L. and Rousseeuw, P. J. (1990). Finding Groups in Data. An Introduction to Cluster Analysis. John Wiley \& Sons, New York.

Linde, Y., Buzo, A., and Gray, R. (1980). An Algorithm for Vector Quantizer Design. IEEE Transactions on Communications, COM-28(1):8495.

Milligan, G. W. and Cooper, M. C. (1985). An examination of procedures for determining the number of clusters in a data set. Psychometrika, 50:159-179.

Porkaew, K., Chakrabarti, K., and Mehrotra, S. (1999). Query Refinement for Multimedia Similarity Retrieval in MARS. In Proceedings of the ACM International Multimedia Conference, Orlando, Florida, US.

Rui, Y., Huang, T., and Mehrotra, S. (1997). Content-based Image Retrieval with Relevance Feedback in Mars. In Proc. of IEEE Int. Conf. on Image Processing '97, pages 815-818.

Sclaroff, S., Taycher, L., and Cascia, M. L. (1997). ImageRover: A Content Based Image Browser for the World Wide Web. In Proc. IEEE Workshop on Content-based Access of Image and Video Libraries. 


\section{Biographies}

Roberto Brunelli was born in Trento, Italy, 1961. He received his degree in physics (summa cum laude) from the University of Trento in 1986. He joined ITC-irst in 1987, where he works in the Computer Vision Group of the Interactive Sensory Systems Division. In the past he was involved in research on computer vision tools, analysis of aerial images, development of algorithms working on compressed description of binary images, optimization, neural networks, and face analysis. His current major involvement is in the area of image similarity retrieval. His current professional interests include optimization, robust statistics, object recognition, and multimedia databases.

Ornella Mich was born in Tesero, Trento, Italy in 1959. She received her degree in electronic engineering from the University of Padova, Italy, in 1987. Then she worked on radar electronics for jet-fighters. After teaching electrotecnics in a high school, she joined ITC-irst in 1989, collaborating on VLSI research. From 1990 she is working in the field of computer vision in the Interactive Sensory Systems Division. 